\title{
Rethinking naive realism
}

\author{
Ori Beck ${ }^{1}$
}

Published online: 12 January 2018

(C) The Author(s) 2018. This article is an open access publication

\begin{abstract}
Perceptions are externally-directed-they present us with a mind-independent reality, and thus contribute to our abilities to think about this reality, and to know what is objectively the case. But perceptions are also internally-dependenttheir phenomenologies depend on the neuro-computational properties of the subject. A good theory of perception must account for both these facts. But naive realism has been criticized for failing to accommodate internal-dependence. This paper evaluates and responds to this criticism. It first argues that a certain version of naive realism, often called "selectionism", does indeed struggle with internal-dependence. It then develops an alternate version of naive realism which does not. This alternate version, inspired by an idea of Martin's, accommodates the internal-dependence of perceptions by recognizing the role that the subject's neuro-computational properties play in shaping perceptual phenomenology. At the same time, it retains the distinctive naive realist account of the external-directedness of perceptions.
\end{abstract}

Keywords Naive realism · Disjunctivism - Perception · Phenomenology · Phenomenal character $\cdot$ Neuro-computational properties

\section{Introduction}

Perceptions combine two interesting features. On the one hand, they present us with mind-independent items, and thus contribute to our abilities to think about those items, and to know what is objectively the case. Following Pautz (2010), we may call this the "external-directedness" of perceptions. On the other hand, the

Ori Beck

ozsb2@cam.ac.uk

1 Christ's College, University of Cambridge, St. Andrews Street, Cambridge CB2 3BU, UK 
phenomenology of perceptions depends on the neuro-computational properties of the subject. Following Pautz (2010), we may call this the "internal-dependence" of perceptions. A good theory of perception must account for both the externaldirectedness and the internal-dependence.

Naive realism is a theory of perception often advertised for its elegant way of accommodating the external-directedness of perceptions. But its critics have complained that naive realism fails to accommodate the internal-dependence of perceptions. Here I respond to this criticism. I first argue that internal-dependence does indeed pose a problem for a specific version of naive realism, called "selectionism". I then develop a different version of naive realism, which is free of the problem. This version of naive realism-called "neuro-computational naive realism"-accommodates internal-dependence by recognizing the role that the subject's neuro-computational properties play in shaping perceptual phenomenology. At the same time, neuro-computational naive realism retains the distinctive naive realist account of the external-directedness of perceptions. ${ }^{1}$

The structure of the paper is as follows: In Sect. 2 I present naive realism and selectionism. In Sect. 3 I explain why the internal-dependence of perceptions poses a problem for selectionism. I discuss and reject selectionist replies in Sects. 4-5. I then develop neuro-computational naive realism in Sect. 6. In the final Sect. 7 I answer a possible objection.

\section{Naive realism and selectionism}

I take naive realism to minimally involve three theses. The first two are more relevant to external-directedness than to internal-dependence, while the third is the other way around. So, since internal-dependence will be our more central concern here, I will mention the first two theses briefly, and then move on to discuss the third.

The first thesis of naive realism, (NR1), states that perceptions are fundamentally experiences in which a subject perceives existing mind-independent items. ${ }^{2}$ This, along with the assumption that hallucinations are not fundamentally experiences in which a subject perceives existing mind-independent items, entails minimal disjunctivism - the view that perceptions and hallucinations are experiences of distinct fundamental kinds. ${ }^{3}$ The second thesis of naive realism, (NR2), states that the $S$ perceives $x$ relation is neither identical to, nor grounded in, representation

\footnotetext{
1 As will become clear later, neuro-computational naive realism extends and develops upon an idea of Martin's (1998). I am deeply indebted to his work.

2 See Brewer (2011, p. 93), Campbell (2002, p. 117), Genone (2016, p. 6), Martin (2002, pp. 392-393, 2004, p. 39, 2006, p. 354) and Snowdon (1990, p. 124).

3 What to say of illusions is trickier; but here I will follow Antony (2011), Brewer (2011), Campbell (in chap. 4 of his and Cassam's 2014), Fish (2009), Genone (2014), Kalderon (2011), and Travis (2013) in taking illusions to be perceptions of some sort. Accordingly, I will henceforth use "perceptions" to include illusions.
} 
relations. ${ }^{4}$ This captures naive realism's opposition to understanding the $S$ perceives $x$ relation in representational terms.

By accepting (NR1) and (NR2), one can accommodate the external-directedness of perceptions. If perceptions fundamentally and non-representationally relate us to existing mind-independent items, then there is a clear sense in which perceptions present us with mind-independent reality. And this, in turn, can arguably explain why perceptions contribute to our abilities to think about this reality, and to know what is objectively the case. In fact, Campbell (2002) and Putnam (1994) have argued that naive realism or similar views are required for an adequate explanation of how we can think about the mind-independent items around us. Relatedly, McDowell (1996, 2008) and Johnston (2006) have argued that views along the lines of naive realism provide us with a distinctive account of perceptual knowledge, which can overcome certain forms of skepticism..$^{5}$

Now turn to the third thesis of naive realism, which concerns perceptual phenomenology. We might think of this thesis as having two versions-a standard version, accepted by all naive realists; and a strengthened version, which is only accepted by those naive realists who are sometimes called "selectionists". 6 In the next section I will argue that the strengthened selectionist version of the thesis runs afoul of the internal-dependence of perceptions. But before I do, I must introduce both versions of the thesis, starting with the standard one.

We may approach the standard version of the third thesis by noting that Brewer (2011, p. 100), Campbell (2002, p. 116), Fish (2009, pp. 49-50), French (2014, pp. 395-396), Logue (2012, p. 212), Martin (1998, pp. 173-175) and other naive realists all hold that the items a subject perceives in having a perception constitutively shape the perception's phenomenology. Importantly, however, naive realists often deny the further claim that only the items a subject perceives in having a perception shape the perception's phenomenology. Campbell (in Campbell and Cassam 2014, chap. 2), for example, suggests that the phenomenology of a perception is in virtue of three distinct factors: (1) the items perceived, (2) the point of view, and (3) the features of the perception relation itself. By "point of view" Campbell (2009, pp. 657-658) means not just the time and place from which one perceives, but a whole set of conditions under which one has the perception. In the case of the visual modality, these conditions include the lighting conditions and the relative orientations and distances the subject is to the perceived items. In the case of other modalities, different conditions might be included. Brewer (2011) has a similar account of points of view; and he suggests that visual perceptual

\footnotetext{
${ }^{4}$ See Brewer (2011, p. 55), Campbell (2002, pp. 117-118), French (2014, p. 395) and Martin (2004, p. 39). Some naive realists-e.g., Travis (2013, p. 31) and Austin (1962, p. 11) - take the stronger view that perceptions do not represent things as being thus and so. But this anti-representationalist view is not a defining commitment of naive realism, and commitment to it is denied, e.g., by French (2014, p. 395).

5 Whether naive realism is the only view that can boast these advantages, and in particular how naive realism compares in these respects with views that deny (NR2), are questions I do not have space to discuss. For a different set of considerations in favor of naive realism, see Martin (2002) and Logue (2012).

${ }^{6}$ See Fish (2009, p. 137) and Pautz (2013, p. 31).
} 
phenomenology is sensitive to the existence of certain similarities, which can be visually relevant relative to one's point of view (see footnotes 21 and 22). With respect to the features of the perception relation itself, Campbell (in Campbell and Cassam 2014, pp. 28, 51) suggests that those can account for the phenomenology of perceiving watchfully or steadily. Similarly, French (2014) suggests that the perceiving relation can have features that account for the phenomenology of perceiving blurrily. Brewer (2011, p. 116) holds that the perceiving relation might be degraded - a feature of the relation which he takes to account for the visual phenomenology enjoyed by a red/green color-blind subject when she visually perceives an apple. Other factors may bear on perceptual phenomenology as well: Fish (2009, chap. 3) writes that the phenomenology of visual perceptions is partly determined by features of the subject's visual system, attention mechanisms and conceptual powers. Campbell (2002, pp. 118-119) similarly recognizes that a subject's neuro-computational properties may bear on her perceptual phenomenology. And Brewer (2011, chap. 5, 2013) suggests that some aspects of visual perceptual phenomenology are sensitive to certain mental episodes of similarity registration.

In view of the diversity of factors (other than the items perceived) that naive realists say bear on the phenomenology of a perception, it will be convenient to have a way of collectively referring to all of them. I will use the term "standpoint". ${ }^{7}$ Let the standpoint of a given perception be the set of all the conditions which naive realists recognize as bearing on the phenomenology of that perception, except for the items perceived. In the case of vision, these conditions include both extradermal conditions of perception (e.g., location and orientation conditions, lighting conditions), as well as conditions of the perceiver's psychological apparatus (e.g., idiosyncrasies of her visual system, attention patterns, and cognitive processing).

With the term "standpoint" so understood, the standard version of the third thesis of naive realism, (NR3), can now be expressed thus: A perception has the phenomenology that it does in virtue of (1) the items perceived in the perception, and (2) the standpoint of the perception. Note that this thesis specifies neither what the perceptual standpoint itself, nor what the perceived items themselves, contribute to the determination of a perception's phenomenology. When it comes to the separate phenomenological contributions of the standpoint and the perceived items, (NR3) is silent.

Since (NR3) is silent on the extent to which standpoints shape perceptual phenomenology, it does not obviously conflict with the internal-dependence of perceptions. In fact, in Sect. 6 I outline how (NR3) can be seen as compatible with internal-dependence. But the same is not true (NR3)'s strengthened version-the version accepted by the selectionist naive realist.

Selectionists-e.g., Fish (2009, pp. 75, 137-138) and Allen (2016, pp. 13-14 and chap. 3) - significantly strengthen (NR3) by adding two further commitments to it. The first is that a perception has the phenomenology that it does completely in virtue of the items perceived in the perception. The second is that standpoint conditions

\footnotetext{
${ }^{7}$ In using this term I follow Campbell (2009).
} 
serve to select which of the many items in the subject's environment the subject perceives. According to selectionism then, the role of standpoints is restricted to the selection of the items perceived. Once selected, these items alone determine the phenomenology of the subject's perception. ${ }^{8}$ For example, when you fix your gaze on a yellow and crescent shaped banana, standpoint conditions may determine that you perceive the banana's being yellow and crescent-shaped. (A banana's being yellow is, on this view, a mind-independent item.) And it is completely in virtue of your perceiving of the banana's being yellow and crescent-shaped that the banana appears yellow and crescent-shaped to you. ${ }^{9}$

Like other forms of naive realism, selectionism does a great job explaining external-directedness. But I will now argue that it cannot explain internaldependence.

\section{Selectionism cannot accommodate internal-dependence: the initial challenge}

According to selectionism, a perception has the phenomenology that it does completely in virtue of the mind-independent items perceived in the perception. This entails that, necessarily, two perceptions of the exact same mind-independent items have the same phenomenology. Unfortunately, however, three empirical arguments put this claim under pressure:

- Attentional variation Empirical results show that phenomenal contrast, saturation, size, spatial frequency, gap size and flicker rate can be modulated by the subject's attention patterns. ${ }^{10}$ In particular, if you present a subject with two identical Gabor patches (grids of sinusoidal luminance stripes) and you make her involuntarily attend to one of them more than to the other, the attended patch would look a higher contrast to her than the unattended one. So following Block (2010), let's suppose you and another normal perceiver look at the same pair of identical Gabor patches under the same normal extra-dermal circumstances. The only difference is that you attend to both patches equally, while your partner (involuntarily) attends more to the right patch. Evidence suggests that the right patch would look different contrasts to each of you. Nevertheless, it is plausible that you both perceive the same items, since you are both normal perceivers placed in the same normal extra-dermal conditions.

\footnotetext{
${ }^{8}$ Some readers may already feel that phenomena such as blurry vision and attention-based modulation of perceptual phenomenology show that selectionism is too strong to be plausible as stated. These are indeed serious concerns; which may well invite the kind of weakened selectionism discussed in Sect. 5. Also see footnote 29.

9 The example is adapted from Logue (2012, p. 211).

10 See Carrasco et al. (2004), Gobell and Carrasco (2005), Ling and Carrasco (2006), Liu et al. (2009) and Montagna et al. (2009).
} 
- Shifted spectra Empirical results show that variations in sex, race and age are associated with variations in the colors that things look to subjects. ${ }^{11}$ So again following Block (2007a), let's suppose you and another normal color perceiver of a different sex, race and age are looking at the same color stimulus under the same extra-dermal conditions. ${ }^{12}$ It is empirically plausible that you would have perceptions with distinct color phenomenology, though you perceive the same items.

- Coincidental variation Empirical results show that similarities in taste, smell, loudness, and color experiences are well correlated with similarities in the experiencer's neuro-computational properties, but poorly correlated with similarities in the perceived properties. ${ }^{13}$ Appealing to this fact, Pautz (2011, 2014, 2017) has argued the following: Suppose you have a twin who belongs to a human-like species. The cross-species similarity ensures that under the same extra-dermal conditions, your and your twin's perceptual systems causally interact with the same environmental items, and exhibit post-receptoral neural response patterns that track the same environmental items to the same optimal degree. Nevertheless, the response patterns themselves are different. When you look at blue things, the distributed neural response of your V4 area resembles the response that area has when you look at purple things but not the response it has when you look at green things. By the empirically-established link between neural similarity and phenomenal similarity, it follows that blue things and purple things look similar in color to you, and that blue things and green things look dissimilar in color to you. On the other hand, when your twin looks at blue things, the distributed neural response of her V4 area resembles the response that area has when she looks at green things but not the response it has when she looks at purple things. By the empirically-established link between neural similarity and phenomenal similarity, it follows that blue things and green things look similar in color to your twin, and that blue things and purple things look dissimilar in color to your twin. Now, since blue things and green things look similar in color to your twin but not to you, and since blue things and purple things look similar in color to you but not to your twin, blue things look different colors to you and to your twin. So consider a particular instance in which you and your twin look at a blue ball under the same normal extra-dermal conditions. By the last argument, that ball looks different colors to you and to your twin. But since the extra-dermal conditions are fixed, and since you and your twin's

\footnotetext{
11 See Hurvich et al. (1968), Neitz and Jacobs (1986), Hardin (1988), Lutze et al. (1990), Neitz et al. (1993) and Neitz and Neitz (1998).

12 The philosophical significance of these cases has also been discussed by Sydney Shoemaker (2000) and Hardin (1988).

13 For correlations regarding taste, see Schiffman and Erickson (1971), Smith et al. (1983), Van der Heijden (1993), Walters (1996), Di Lorenzo et al. (2009), Sakurai et al. (2010) and Chen et al. (2011). For those regarding smell, see Malnic et al. (1999), Cowart and Rawson (2005), Youngentob et al. (2006), Margot (2009) and Howard et al. (2009). For those regarding loudness, see Röhl et al. (2011), Langers et al. (2007), Moore (2003) and Relkin and Doucet (1997). Finally, for those regarding color, see MacAdam (1985) and Brouwer and Heeger (2013).
} 
perceptual systems optimally track the same environmental items, you plausibly both perceive the exact same items.

We now have three cases in which two subjects have perceptions that differ in color or contrast phenomenology, even though the subjects are under identical extradermal conditions. This suggests that perceptions are internally-dependent, i.e., that perceptual phenomenology depends on the neuro-computational properties of the subject. Furthermore, in each of the cases the subjects plausibly perceive the same items. So we seem to also have cases in which the identity of the perceived items is conjoined with distinctness in perceptual phenomenology. If so, selectionism is refuted.

Faced with these arguments, a selectionist could offer one of two responses: the subjects perceive different mind-independent items, or the subjects in fact have perceptions with the same phenomenology. I will argue that neither response works. Then I will suggest a quite different form of naive realism.

\section{Response 1: Different items perceived?}

The first selectionist response is to say, in a given case, that the two subjects perceive different mind-independent items. But what might these different items be?

A natural answer would be that the mind-independent colors one perceives determine one's perceptual color phenomenology, and that the mind-independent contrasts one perceives determine one's perceptual contrast phenomenology. Differences in perceptual color phenomenology are therefore due to differences in the colors one perceives, while differences in perceptual contrast phenomenology are due differences in the contrasts one perceives. If we apply this to our cases, we will say that since the subjects in the shifted spectra and coincidental variation cases have perceptions that differ in color phenomenology, they perceive different colors. Similarly, since the subjects in the attentional variation case have perceptions that differ in contrast phenomenology, they perceive different contrasts.

This selectionist line of thought can be further articulated in several ways: (1) Fish (2009, pp. 165-177) suggests that certain cognitive and optical illusions occur when psychological processes bring about two effects simultaneously. First, the subject is prevented from perceiving a certain mind-independent item, which she would otherwise have perceived. Second, the subject comes to believe that a different item is (or, seems to be) there. This idea can be applied in our cases. In any one of them, we could say that one subject perceives the color or contrast that is before her. The other subject (because of some psychological reason) does not perceive any color or contrast. Nevertheless, the subject believes that some other color or contrast is (seems to be) there. (2) Here is an alternative suggestion. Let's say that two color or contrast properties overlap just in case their degrees of determinacy allow them to be co-instantiated. For example, being blue (being $22 \%$ in contrast) and being a color between bluish-green and bluish-red (being 16-28\% in contrast) are overlapping properties. Similarly, being a color between bluishgreen and reddish-blue (being 16-23\% in contrast) and being a color between 
greenish-blue and bluish-red (being 21-28\% in contrast) are overlapping properties. Using this terminology, we can suggest that in any one of our cases, the subjects perceive distinct but overlapping color or contrast properties. These differences account for the differences in their perceptual color or contrast phenomenologies. ${ }^{14}$ (3) Byrne and Hilbert (1997) favorably consider the proposal that objects can be both unique green (i.e., green that is neither yellowish nor bluish) and bluish green all over and at the same time. They develop this proposal by first suggesting that colors are, roughly, types of surface spectral reflectances. They then suggest that such types might intersect. In particular, the surface spectral reflectance type identical to unique green might intersect with the surface spectral reflectance type identical to bluish green. In that case, some surface spectral reflectance would belong to both types; and any object which had that surface spectral reflectance would be both unique green and bluish green. Now, while Byrne and Hilbert are not selectionists themselves, a selectionist could use their proposal to account for cases in which a single object looks unique green to one normal subject but bluish green to another normal subject, despite the fact that both subjects are under fixed extradermal conditions. The account would be that for some reason, one subject perceives the unique greenness of the object; while the other perceives its bluishgreenness. Interestingly, Fish considers a related suggestion in his (2009, p. 154, footnote 3$)$.

Though all these suggestions are worthy of consideration, Pautz's coincidental variation case poses a particularly serious problem for them. This is because Pautz explicitly designed the coincidental variation case to rule out the possibility that the subjects in the case perceive different items. As we noted in Sect. 3, Pautz rules out this possibility by stipulating that the perceptual systems of the two subjects in the case optimally track the same environmental items. Given this stipulation, the "different items perceived" response (in any of its forms) appears implausible for the coincidental variation case.

I doubt, however, that selectionists will accept this assessment of the situation; and in fact, some have already resisted it. In particular, Allen (2016, pp. 65-73) has insisted that in a version of the coincidental variation case in which a single ball appears blue to you but green to your twin, you and your twin perceive completely different colors of the ball. According to Allen, this is possible because objects can have multiple mind-independent colors (and, more broadly, multiple mindindependent qualitative natures) at a time. Furthermore, Allen denies that if subjects' perceptual systems optimally track the same items, then they perceive the same colors. According to him, even if you and your twin's perceptual systems optimally track the same items, you two may still perceive different colorsassuming that you two also enjoy different post-receptoral neural response patterns.

Though Allen's proposal is ingenious, I do not believe it can be accepted; and I will now argue, with Pautz, that the subjects in the coincidental variation case

\footnotetext{
14 James Stazicker (2011) discusses the interaction between visual indeterminacy and attention. I am grateful to him for a discussion of this alternative.
} 
perceive the same colors. I will do so by focusing on a particular instance of the coincidental variation case.

Before I introduce the instance of the coincidental variation case I have in mind, let's recall two empirical results concerning human color perception. The first is that normal color perceivers enjoy color constancy: To a remarkable extent, they perceive stable colors over a wide range of illumination conditions. Though the neural mechanisms responsible for color constancy are not fully understood, there is significant evidence that the phenomenon is (at least in part) due to post-receptoral neural response patterns that recover object colors from the retinal input (which confounds these colors with features of the illumination). ${ }^{15}$ This suggests, at least on the assumption that colors are perceptible mind-independent items, that there are post-receptoral neural response patterns that track perceived colors. The second result is that the post-receptoral neural response patterns which track colors causally enable normal color perceivers to (a) discriminate between areas that are different (even if similar) colors, (b) attend to colored areas, (c) visually guide various actions on colored areas, and (d) recognize colored areas as being the colors that they areall in the service of the goals of the perceivers. ${ }^{16}$ Equipped with these empirical results, we can consider the following instance of Pautz's coincidental variation case:

- Coincidental variation* Suppose you are a normal color perceiver, who has a twin belonging to a human-like species. The cross-species similarity ensures three things: First, under the same extra-dermal conditions, your and your twin's perceptual systems causally interact with the same environmental items, and exhibit post-receptoral neural response patterns that track the same environmental items to the same optimal degree. Second, like you, when your twin perceives a color, her post-receptoral neural response patterns track that color. Third, like you, when your twin's post-receptoral neural response pattern tracks a color, that tracking causally enables her to (a) discriminate between the area that is that color and nearby areas that are different (even if similar) colors, (b) attend to the area that is that color, (c) visually guide various actions on the area that is that color, and (d) recognize the area that is that color as being that color-all in the service of her goals. Nevertheless, your and your twin's neural response patterns are themselves different. When you look at blue things, the distributed neural response of your V4 area resembles the response that area has when you look at purple things but not the response it has when you look at green things. When your twin looks at blue things, the distributed neural response of her V4 area resembles the response that area has when she looks at green things but not the response it has when she looks at purple things. Now consider a particular instance in which you and your twin look at a blue ball under the same normal extra-dermal conditions.

\footnotetext{
15 See Brouwer and Heeger (2009), Foster (2011) and Roe et al. (2012).

16 See Hardin (1988), Brouwer and Heeger (2013) and Roe et al. (2012).
} 
I will argue that in this coincidental variation* case, the blue ball looks different colors to you and to your twin. At the same time, both you and your twin perceive the same colors.

The argument that the blue ball looks different colors to you and to your twin is familiar from Sect. 3: When you look at the blue ball, the distributed neural response of your V4 area resembles the response that area has when you look at purple things but not the response it has when you look at green things. By the empirically-established link between neural similarity and phenomenal similarity, it follows that the blue ball and purple things look similar in color to you, and that the blue ball and green things look dissimilar in color to you. On the other hand, when your twin looks at the blue ball, the distributed neural response of her V4 area resembles the response that area has when she looks at green things but not the response it has when she looks at purple things. By the empirically-established link between neural similarity and phenomenal similarity, it follows that the blue ball and green things look similar in color to your twin, and that the blue ball and purple things look dissimilar in color to your twin. Now, since the blue ball and green things look similar in color to your twin but not to you, and since the blue ball and purple things look similar in color to you but not to your twin, the blue ball looks different colors to you and to your twin.

It remains to argue that both you and your twin perceive the same colors. To see why this is so, suppose (for reductio) that as you look at the blue ball, you and your twin perceive different colors. If you perceive different colors, there is a color $C$ that is perceived by one of you, but not by the other. Consider the subject that does perceive $C$. By the stipulations of the case, that subject-whether she is human or merely human-like - has a post-receptoral neural response pattern that tracks color $C$. The stipulations also say that both you and your twin are under the same extradermal conditions; and that when you two are under the same extra-dermal conditions, you both have post-receptoral neural response patterns that track the same environmental items. So if one of you has a post-receptoral neural response pattern that tracks color $C$, the other does as well. It follows that both you and your twin have post-receptoral neural response patterns that track color $C$. Finally, the case's stipulations say that when you and your twin have post-receptoral neural response patterns that track a color, that tracking causally enables both of you to (a) discriminate between the area that is that color and nearby areas that are different (even if similar) colors, (b) attend to the area that is that color, (c) visually guide various actions on the area that is that color, and (d) recognize the area that is that color as being that color-all in the service of your respective goals. Therefore, your and your twin's tracking of color $C$ causally enable both of you to (a) discriminate between the area that is $C$ and nearby areas that are different (even if similar) colors, (b) attend to the area that is $C$, (c) visually guide various actions on the area that is $C$, and (d) recognize the area that is $C$ as being $C$-all in the service of your respective goals.

Now consider the following principle:

(P) If (i) $S$ 's visual perception system exhibits a post-receptoral neural response pattern that tracks a color $C$, and (ii) that tracking causally enables $S$ to 
(a) discriminate between the area that is $C$ and nearby areas that are different (even if similar) colors, (b) attend to the area that is $C$, (c) visually guide various actions on the area that is $C$, and (d) recognize the area that is $C$ as being $C$-all in the service of $S$ 's goals, then $S$ visually perceives color $C$.

Principle $(\mathrm{P})$ is supported by the results and methods of perceptual psychology. Perceptual psychology is a fruitful and quickly advancing science. This science seeks to explain the perception of various kinds of items by finding mechanisms by which such items are tracked, and by showing how the tracking contributes to the perceivers' abilities to perform tasks that perceivers of these items can distinctively perform for the advancement of their goals. ${ }^{17}$ Perceptual psychology has had significant successes in doing this for color perception. ${ }^{18}$ Though the full details of color perception are still far from fully understood, existing results do suggest that a subject $S$ 's perception of $C$ is explained by the truth of (P)'s antecedent. And if the truth of (P)'s antecedent explains $S$ 's perception of $C$, then $(\mathrm{P})$ is itself true.

Now apply principle $(\mathrm{P})$ to the coincidental variation* case. We have already established that the case is one in which both you and your twin satisfy (P)'s antecedent with respect to color $C$. Therefore, both you and your twin perceive color $C$. But, ex hypothesi, $C$ is a color that is perceived by one of you, but not by the other. Contradiction. It follows that, for any color $C$, either you and your twin both perceive it, or neither of you do. Furthermore, since you and your twin have perceptions with different color phenomenologies, the selectionist suggestion that the mind-independent colors one perceives determine one's perceptual color phenomenology is false.

At this point, selectionists might make a partial retreat. They might concede that the mind-independent colors one perceives do not determine one's perceptual color phenomenology. But they might still insist that some mind-independent items that one perceives do determine one's perceptual color phenomenology. They might also insist that the subjects in the coincidental variation* case do not perceive exactly the same mind-independent items. Thus, they might try to retain selectionism in the face of the coincidental variation* case.

This selectionist response requires that some mind-independent items that one perceives determine one's perceptual color phenomenology. The trouble is that it is difficult to see what these items might be. To illustrate the difficulty, I will consider three possibilities.

First, consider the possibility that the mind-independent visually basic properties that one perceives determine one's perceptual color phenomenology. By "visually basic properties" I have in mind, in addition to colors, also properties like sizes, shapes, visible textures and spatial arrangements. ${ }^{19}$ This possibility is unattractive

\footnotetext{
17 See Marr (1982) and Palmer (1999).

18 In addition to the aforementioned results, see Conway et al. (2010).

19 It was suggested to me that Martin motivates this view in his (2010). I disagree. In (2010) Martin argues for the "parsimonious" view that "objective looks" can be identified with visually basic properties. He (2010, pp. 220-221) takes this identification to suggest that a straight stick in open air and a straight stick half submerged in water have the same objective looks. And he further appeals to such
} 
for two reasons: (1) The perception of visually basic properties is-like the perception of colors-plausibly explainable by appeal to neural responses which track these properties and then enable subjects to perform distinctive tasks for the advancement of their goals. Therefore, arguments using principles analogous to (P) could plausibly show that subjects in cases like the coincidental variation* case perceive the same visually basic properties. If so, those cases would constitute counterexamples to the thesis that the mind-independent visually basic properties one perceives determine one's perceptual color phenomenology. (2) We know from the coincidental variation* case that the perception of a ball's color does not determine the perceptual color phenomenology associated with the ball. We also know-from our own experiences - that the perception of a ball's size, shape, visible texture or spatial arrangement does not determine the perceptual color phenomenology associated with the ball. Together, these two data points make it highly implausible that the perception of any subset of a ball's visually basic properties determines the perceptual color phenomenology associated with the ball. So, again, it appears that the visually basic properties one perceives do not determine one's perceptual color phenomenology.

Second, consider the possibility that subjects normally perceive their own neurocomputational properties; and that the neuro-computational properties that one perceives determine one's perceptual color phenomenology. This possibility is unattractive simply because it is implausible that we normally perceive our own neuro-computational properties. Visual perception requires there to be a causal chain which leads from the perceived item to the eyes, and from there to the subject's visual cortex. But normally, there is no such chain leading from our neurocomputational properties to our eyes and from there to our visual cortex. More importantly, visual perception usually enables us to visually discriminate the perceived item from the rest of the environment. But normally we cannot so discriminate our neuro-computational properties. Lastly, visual perception usually enables us to attend to the perceived items. But, again, we normally cannot so attend to our neuro-computational properties.

Third, consider suggestions made by Fish (2009, pp. 150-161) and Genone (2014). According to them, objects can have relational properties of (roughly) the kind "being color $C$, under such and so illumination, and in such and so surround". Call these properties "relational colors". Fish and Genone hold that when normal subjects are normally presented with such relational colors, the subjects perceive these relational colors. And the relational colors subjects perceive determine (at least the most specific features of) the subjects' perceptual color phenomenology.

\footnotetext{
Footnote 19 continued

objective looks in offering a "minimalist" account of statements about how things look (to $S$ ). On this account, these statements often involve implicit comparisons between the objective looks of objects or the sensory episodes of subjects on the one hand, and contextually relevant paradigm cases on the other. Martin insists that this account is minimal, because it "commits us hardly at all to the underlying nature of sense experience. ...And indeed, the point of the account ... is not to offer grounds for preferring one theory of sensory experience over another" (2010, pp. 222-223). In view of this, I think that one would be over-reading Martin's paper were one to take it to be a defense of selectionism. I will return to Martin's views in Sect. 6.
} 
For example, here is how Fish and Genone explain why white things look different shades to people when under white and yellow lights: Under white lights, white things have "whitish" relational colors; while under yellow lights they have "yellowish" relational colors. When normal subjects are normally presented with whitish relational colors, they perceive the whitish relational color. So things look whitish to them. When normal subjects are normally presented yellowish relational colors, they likewise perceive the yellowish relational colors. So things look yellowish to them. Thus, white things look different shades to people when under white and yellow lights.

It appears, however, that Fish and Genone's position cannot provide a selectionist-friendly treatment of the coincidental variation* case. In the coincidental variation* case, both subjects are looking at the same scene, under the same extra-dermal conditions. In particular, they are looking at the same blue ball, under the same illumination and in the same surround. So they are facing the same relational colors. Furthermore, the subjects perceive the same relational colors. This is because, according to Fish and Genone, when normal subjects are normally presented with relational colors, they perceive them. And in the coincidental variation case, both subjects are normal, and normally presented with the same relational colors. We thus have a case in which two subjects perceive the same relational colors, though their perceptions have different color phenomenologies. So it cannot be that the relational colors one perceives determine one's perceptual color phenomenology.

In defense of Fish and Genone, one might propose that the properties that are relevant to determining one's perceptual color phenomenology are not the relational colors Fish and Genone discuss, but something similar. In particular, one might propose that objects have relational colors*-properties of (roughly) the kind "being color $C$, under such and so illumination, in such and so surround, and causing the instantiation of such and so neuro-computational properties". It is the perception of these relational colors* that determines one's perceptual color phenomenology.

This proposal has the advantage of being immune to the argument in the previous paragraph: Ex hypothesi, the subjects in the coincidental variation* case have different neuro-computational properties. Given the definition of relational colors*, this suggests the subjects also face different relational colors*.

Unfortunately, however, the proposal also has a disadvantage. I argued earlier that we do not normally perceive our own neuro-computational properties. It is even clearer that we do not normally perceive the neuro-computational properties of others. So, in general, we do not normally perceive any neuro-computational properties. A fortiori, we do not normally perceive colors' causing instantiations of any neuro-computational properties. Much less do we normally perceive colors', under given illumination conditions and in given surround conditions, causing instantiations of any neuro-computational properties. And this, given the definition of relational colors*, suggests that we do not normally perceive relational colors*. 


\section{Response 2: Same phenomenology?}

An alternative way of defending selectionism is to suggest, with respect to at least some of our cases, that the subjects' perceptions have the same phenomenology.

Stated so strongly, this alternative defense of selectionism is flatly contradicted by aforementioned empirical findings. But it turns out that if selectionism is somewhat weakened, a more sophisticated version of this defense becomes available.

The weakened version of selectionism concedes that some aspects of phenomenology are not completely in virtue of the items the subject perceives. So it is no longer necessarily true that if two subjects perceive the same items, their perceptions have the same phenomenology. Nevertheless, the view is still somewhat selectionist, as it insists that there are aspects of phenomenology (call them "core phenomenal aspects") which are completely in virtue of the items the subject perceives. The suggestion is thus that $S$ 's standpoint plays the role of selecting the items that $S$ perceives, and that the core phenomenal aspects of the subject's perception are completely in virtue of those items. Thus, if two subjects perceive the same items, though their perceptions may differ in some phenomenal ways, the perceptions will necessarily have the same core phenomenal aspects.

This weakened selectionism can be used to accommodate our cases in two ways. First, one might concede (in a given case) that things look distinct colors/contrasts to the subjects, but deny that this constitutes a difference in the core phenomenal aspects of perception. Alternatively, one might insist (in a given case) that things look the same color/contrast to both subjects; and that the empirically verified phenomenal differences between them concern other, non-core, aspects.

The first of these two suggestions is unattractive. To see this, consider the robustness of the empirical results driving the attentional variation, shifted spectra and coincidental variation cases. Put together, these results imply that it is possible for two subjects to perceive the same items, while their perceptions differ not just color or contrast phenomenology, but also in saturation, size, spatial frequency, gap size, flicker rate, taste, smell or loudness phenomenology. If we answer all the putative counterexamples to selectionism along the lines suggested by the first suggestion, we will be saying that the phenomenal aspects associated with color, contrast, saturation, size, spatial frequency, gap size, flicker rate, taste, smell and loudness are not core phenomenal aspects. In that case, it is unclear what phenomenal aspects of perception would be left over for selectionism to account for. At best, the remaining set of core phenomenal aspects would be a meager and disjointed one. And though a version of selectionism that can only account for those phenomenal aspects is not without interest, I think we should at least pause before accepting it. $^{20}$

Consider the second suggestion next. To review, the suggestion is that both subjects (in a given case) enjoy the same color or contrast phenomenology; and that phenomenal differences between them concern non-core aspects. Interestingly,

${ }^{20}$ Cf. Logue $(2012,2017)$. 
Brewer (2013) has developed a response along these exact lines for the attentional variation case.

Somewhat amended to fit with weakened selectionism, Brewer's (2011, chap. 5, 2013) view is as follows: There are ways that objects thinly look to us, and ways that objects thickly looks to us. An object $o$ thinly looks $F$ to $S$ iff $S$ perceives $o$ 's being $F$-like. This suggests that objects thinly look to us as they do completely in virtue of the items we perceive. Therefore, ways objects thinly look to us count as core phenomenal aspects. ${ }^{21}$ At the same time, an object $o$ thickly looks $F$ to $S$ iff $o$ thinly looks $F$ to $S$, and $S$ mentally registers that $o$ is $F$-like. ${ }^{22}$ This suggests that objects do not thickly look to us as they do completely in virtue of the items we perceive. Ways objects thickly look to us are thus not core phenomenal aspects.

Now in the attentional variation case, Brewer (2013) suggests that the subjects' perceptions have the same core phenomenal aspects, since the Gabor patch thinly looks exactly the same to both of them. But the patch thickly looks different contrasts to them, because the subjects' different attention patterns lead each of them to mentally register different things.

Brewer's account of the case is ingenious. On the one hand, it appears consistent with the empirical results, since it describes the subjects as enjoying different ("thick") contrast phenomenologies. On the other hand, the account brings out a way of preserving a weakened version of selectionism.

Unfortunately, Brewer's account cannot be accepted. The key problem concerns Brewer's position on thick looks. It entails that if $o$ thickly looks $F$ to $S$, then $o$ also thinly looks $F$ to $S$. Now in the attentional variation case, Brewer says that the Gabor patch thickly looks different contrasts to the subjects. So he must also say that the patch thinly looks different contrasts to them. How is this consistent with his suggestion that the patch thinly looks exactly the same to them?

Brewer $(2011$, p. 125, 2013) attempts to answer this question. He notes that white chalks in red light simultaneously thinly look both red and white-and-in-red-light to perceivers. This shows that objects can simultaneously thinly look multiple ways to perceivers. If so, there is no problem in suggesting that in the attentional variation case, the Gabor patch simultaneously thinly looks two distinct contrasts to the subjects. In thinly looking both these contrasts to both subjects, it thinly looks exactly the same to them. Nonetheless, if one subject registers only one contrastrelated feature of the patch, while the other registers only the other contrast-related feature of the patch, the patch would thickly look distinct contrasts to each of them.

But this answer is misleading, as it fails to distinguish between competing and non-competing looks. I say that $F$ looks and $G$ looks compete iff nothing could

\footnotetext{
${ }^{21}$ Brewer's unamended suggestion is that $o$ thinly looks $F$ to $S$ iff $S$ is acquainted with $o$ from a point of view relative to which $o$ has visually relevant similarities to paradigm $F$ s; where a paradigm $F$ is a possible $F$ object which we (qua English speakers) associate with the term " $F$ " in understanding the term. This entails that objects thinly look to us as they do partly in virtue of certain unperceived similarity relations the objects bear to certain paradigms. So on Brewer's unamended suggestion, ways objects thinly look to us do not count as core phenomenal aspects. Also see footnote 22 .

22 Brewer's unamended suggestion is that $o$ thickly looks $F$ to $S$ iff $o$ thinly looks $F$ to $S$, and $S$ mentally registers its visually relevant similarities to paradigm $F \mathrm{~s}$; where the registration can occur either by an active application of the concept $F$, or by imagistic or behavioral means. Also see footnote 21 .
} 
simultaneously appear both $F$ and $G$ to a normal perceiver under normal conditions. A red look and a white-in-red-light look are not competing looks because white chalks in red light do look like both red-chalks-in-white-light and white-chalks-inred-light to normal perceivers under normal conditions. In contrast, a red look and a white look are competing looks, because nothing could simultaneously look both red and white to a normal perceiver under normal conditions.

The notion of competing looks places a plausibility constraint on "thin looks" claims. It is implausible to claim that a white chalk in red light simultaneously thinly looks both red and white to people, if nothing could simultaneously look both red and white to them. And generally, it is implausible to claim that $o$ thinly looks both $F$ and $G$ to normal perceivers in normal conditions, if an $F$ look and a $G$ look are competing looks. Claims that violate this constraint cannot be taken as plausible descriptions of normal experiences. They could perhaps be taken as pieces of philosophical theorizing, but in that case they would shed little light on normal phenomenology.

Now return to the attentional variation case, where a patch looks distinct contrasts to distinct subjects. For concreteness, say it looks $18 \%$ contrast to one, and $22 \%$ contrast to the other. If Brewer's account of the case is to succeed, the patch must thinly look both 18 and $22 \%$ contrast to both. But this claim violates our constraint. Nothing can simultaneously look both 18 and $22 \%$ contrast to a normal perceiver under normal conditions. So Brewer's account fails.

One might offer two responses on Brewer's behalf. The first is to describe the case as involving variable degrees of phenomenal determinacy. E.g., perhaps the patch thinly looks both a determinate $22 \%$ and an indeterminate $16-28 \%$ contrast to both perceivers, and thickly looks only one of these ways to each. This description does not violate the plausibility constraint, assuming a patch can simultaneously look both 22 and 16-28\% contrast to a normal perceiver under normal conditions. However, as Block (2010) argues, the experimental results clearly suggest that the patches look equally determinate ways to both perceivers. So the first response fails.

The second response draws on Brewer's discussion of the shifted spectra case (2011, pp. 127-128). The discussion is difficult, but on one reading it suggests the following: In the shifted spectra case, a color chip thinly looks a single color to the two subjects. Nevertheless, because they understand color terms differently, each of them registers the color-related features of the chip by employing distinct concepts. Therefore, the chip thickly looks different colors to them. In the attentional variation case, one could similarly suggest that the patch thinly looks a single contrast to the subjects. Nevertheless, because they understand contrast terms differently, they each register the contrast-related features of the patch by employing distinct concepts. Therefore, the patch thickly looks different contrasts to them.

This response is also unsuccessful. We can stipulate (in both the shifted spectra and attentional variation cases) that the subjects understand color/contrast terms in exactly the same way (or in no way at all). This stipulation does not disturb the empirical evidence suggesting that things look distinct colors/contrasts to the subjects.

Moving beyond Brewer's view, let's ask if there is some other way of arguing that both subjects (in a given case) have perceptions with the same color or contrast 
phenomenology. Here's a general reason to say "no": The shifted spectra and attentional variation cases are based on findings gathered using methods designed to assess color and contrast phenomenology. These methods have produced a great deal of evidence most readily explained by hypothesis $(\mathrm{H})$ :

(H) When placed in the relevant conditions, the subjects' perceptions have different color or contrast phenomenologies.

The view that both subjects (in either of the cases) have perceptions with the same color or contrast phenomenology, however, denies $(\mathrm{H})$. Instead, it accepts hypothesis $(\neg \mathrm{H})$ :

$(\neg \mathbf{H})$ When placed in the relevant conditions, the subjects' perceptions have the same color or contrast phenomenology.

Consequently, this view must predict that any reliable test of color or contrast phenomenology would produce very different evidence than what we in fact have. This does not mean that the view cannot accommodate the evidence we do have. It can indeed explain such evidence by appealing to some further hypothesis. But the point is that the view cannot predict the evidence without a further complicating hypothesis. And in failing to predict the available evidence without the postulation of further mechanisms, the view loses much of its plausibility. It is bad methodology to accept it.

A similar point applies in the coincidental variation case. If the subjects in that case have perceptions with the same color phenomenology, then similarities in their perceptual color phenomenology do not correlate with similarities in their distributed neural responses. But empirical evidence suggests that in actual cases such similarities are correlated. Therefore, to suggest that subjects in the coincidental variation case have perceptions with the same color phenomenology is to also suggest that the observed actual correlation is merely coincidental. And that is very implausible. As Pautz (2017, p. 31) originally put it, the suggestion has "the absurd implication that it is just a fluke that in normal humans like yourself there is quite generally a nice agreement between neural similarity and qualitative similarity ("good internal correlation")—-somewhat as it would be a fluke if it turned out that similar-looking names (e.g., "Ned" and "Fred") always named similar-looking individuals."

\section{Neuro-computational naive realism: a way of accommodating external-directedness and internal-dependence}

We can draw two lessons from the previous discussion. The first is that perceptions are internally-dependent, i.e., their phenomenologies depend on the neuro-computational properties of the subject. This lesson is particularly evident from the empirical results at the basis of the coincidental variation case. To repeat, the results are that there is a strong correlation between neural similarity and phenomenal similarity. This correlation plausibly suggests that by determining the similarity relations between your actual neural activation and related possible neural 
activations, we can completely determine what colors things look to you, how loud things sound to you, as well as how things smell or taste to you. The second lesson from the previous discussion is that selectionism is false. If we wish to be naive realists, we should be non-selectionist naive realists.

The rest of this paper is an attempt to take these two lessons seriously. More specifically, it is an attempt to outline a form of naive realism that is both nonselectionist, and that acknowledges that perceptions are internally-dependent in the ways suggested by empirical findings. I call this form of naive realism "neurocomputational naive realism".

Neuro-computational naive realism is inspired by Martin's (1998, pp. 173-175) important idea that

to have an experience is to have a viewpoint on something: experiences intrinsically possess some subject-matter which is presented to that viewpoint. To understand such experience and what it is like, one has to understand the viewpoint on that subject-matter, and hence also to attend to the subject matter as presented to the viewpoint. ... On this view, difference in presented elements between two experiences will be sufficient for difference in their phenomenal properties.

Immediately following this passage, Martin (1998, p. 175) adds a remark on the "much stronger" thesis that "sameness and difference of phenomenal properties just are sameness and difference in presented elements":

It is doubtful that this claim is true: why cannot the ways in which things are presented in experience make a difference to what the experience is like, in addition to what is presented?

Martin is suggesting that aspects of perceptual phenomenology are not mere presentations of items. Rather, they are presentations of items in certain ways. In other words, Martin accepts a "multiple component" thesis, according to which aspects of perceptual phenomenology have both a presented item component and a way of presentation component. This entails that perceptions presenting distinct items will be perceptions with distinct phenomenologies. But it also allows perceptions of the same items to differ in their phenomenologies, when the items are presented to the perceiver in different ways.

I wish to elaborate Martin's multiple component thesis by making three suggestions. First, I distinguish between (1) items that perceptually appear to subjects, (2) subjects' appearance properties and (iii) the perceptual appearance relation. The items that perceptually appear to subjects broadly correspond to Martin's presented items. So, for example, a particular ball can perceptually appear to you various ways. So can its size or shape. Appearance properties, on the other hand, broadly correspond to Martin's ways of presentation. To have an appearance property is to be appeared to in a certain way. For example, you can be appeared to 
in a roundish way, or in a bluish way. ${ }^{23,24}$ Finally, the perceptual appearance relation is the three place relation $x$ perceptually appears $W$ to $S$. This is a relation that obtains between a subject $S$, an appearance property $W$ that $S$ instantiates, and an item $x$. When $S$ stands in this relation to some item $x$ and to some appearance property $W$, not only is $S$ appeared to in some way, but furthermore, $x$ perceptually appears that way to $S$. For example, when you stand in this relation to both a particular ball and the appearance property of being appeared to in a roundish way, not only are you roundishly appeared to, but furthermore, the ball perceptually appears round to you. Note that since both perceptually appearing items and appearance properties are relata of the $x$ perceptually appears $W$ to $S$ relation, instantiations of this relation have perceptually appearing items and appearance properties as constituents. Thus, when the $x$ perceptually appears $W$ to $S$ relation is instantiated in the ball's perceptually appearing round to you, the constituents of this instantiation are yourself, your property of being appeared to in a roundish way, and the ball.

My second suggestion is that the relation $x$ perceptually appears $W$ to $S$ is intimately connected to the relation $S$ perceives $x$. The two relations are connected by the fact that, necessarily, if $x$ perceptually appears $W$ to $S$, then $S$ perceives $x$. For example, if a ball appears round to you, you must perceive that very ball. This ensures that perceived items shape conscious perceptual phenomenology, as (NR3) requires. It also ensures that subjects who consciously perceive distinct items will thereby have perceptions with distinct phenomenologies.

My third suggestion concerns appearance properties. What determines which appearance property $W$ a subject instantiates at a time? Here is the appropriate place to consider the aforementioned empirical results. Properly construed, these results suggest that subjects have the appearance properties that they do completely in virtue of their standpoints, and in particular, completely in virtue of their neurocomputational properties. ${ }^{25}$ In other words, your neuro-computational properties completely determine the ways you are appeared to. ${ }^{26}$ But note: To say that your neuro-computational properties completely determine your appearance properties is not to say that your neuro-computational properties completely determine your

\footnotetext{
${ }^{23}$ How ways of being appeared to should be specified is, of course a vexed question (see, e.g., Block 2007b). Happily, for our purposes it is enough to note that by " $S$ is being appeared to in a roundish way" I roughly mean that $S$ is appeared to in the way that I would be appeared to were I to now be confronted by a round thing under ideal conditions.

24 My use of "appearance properties" is different from Genone (2014), Antony (2011), Kalderon (2011), Shoemaker $(1994,2000,2006)$ and others' use of "appearance properties". On their use of the term, appearance properties are not properties that subjects can have, but properties that objects or scenes can have. More specifically, appearance properties are ways that objects or scenes appear simpliciter (as opposed to ways that they appear to a specific subject). This use of "appearance properties" should be carefully distinguished from my own.

25 Note, however, that these properties are probably not restricted to the properties of a particular brain area. Area-restricted properties can be instanced in a collection of cells growing in a bottle, and those cells would presumably not be phenomenally conscious.

26 Of course, external events may cause you to have certain neuro-computational properties. In so doing, external events may play a causal role in the instantiation of appearance properties.
} 
perceptual phenomenology. Perceptual phenomenology is fixed by instantiations of the $x$ perceptually appears $W$ to $S$ relation. Appearance properties are among the relata of this relation, and distinct from it. For example, a ball's perceptually appearing round to you is one thing, and your being appeared to in a roundish way is another. Your neuro-computational properties determine the latter. The former further requires you to perceive the ball.

These three suggestions, taken together with (NR1)-(NR3), constitute neurocomputational naive realism. Neuro-computational naive realism accounts for perceptual phenomenology thus: Suppose a ball $(x)$ perceptually appears round $(W)$ to you $(S)$. That fixes an aspect of your perceptual phenomenology. Consistently with (NR3), neuro-computational naive realism holds that you enjoy this aspect in virtue of your perceiving the ball from a certain standpoint, while having a certain neuro-computational property. Had you failed to perceive the ball, it would not have perceptually appeared round to you, and your perceptual phenomenology would have been different. Similarly, had you been in a suitably different neurocomputational state, you would not have had the same appearance properties, and the ball would also not have perceptually appeared round to you. So, again, your perceptual phenomenology would have been different.

Neuro-computational naive realism has three distinct advantages:

First, neuro-computational naive realism resolves the worry with which this paper began. The worry was that naive realism is in tension with the fact that perceptions are internally-dependent (i.e., their phenomenologies depend on the neuro-computational properties of the subject). The response is that although selectionist naive realism is in tension with the internal-dependence of perceptions, neuro-computational naive realism is not. According to neuro-computational naive realism, the phenomenologies of perceptions consist of instantiations of a three place relation: $x$ perceptually appears $W$ to $S$. The second relatum of this relation is determined by the subject's neuro-computational properties. Therefore, the phenomenologies of perceptions are partially determined by the subject's neurocomputational properties. There is thus no tension between neuro-computational naive realism and the fact that perceptions are internally-dependent. At the same time, the first relatum of the $x$ perceptually appears $W$ to $S$ relation is a perceived mind-independent item. Therefore, the phenomenologies of perceptions are partially determined by the mind-independent items that the subject perceives. Perceived items thus participate in the shaping of perceptual phenomenology.

Importantly, neuro-computational naive realism accommodates the internaldependence of perceptions while simultaneously accommodating the externaldirectedness of perceptions (i.e., the fact that perceptions present us with a mindindependent reality). Neuro-computational naive realism accommodates this external-directedness by accepting (NR1) and (NR2). Together, these theses suggest that perceptions fundamentally and non-representationally relate us to existing mind-independent items. So there is a clear sense in which perceptions present us with mind-independent reality.

The second advantage of neuro-computational naive realism is that it is consistent with the attentional variation, shifted spectra, coincidental variation, and coincidental variation* cases. In each of them, it is either an empirical fact or an 
explicit stipulation that the subjects have different neuro-computational properties. Since the subjects have different neuro-computational properties, it is consistent with neuro-computational naive realism that the subjects have different appearance properties as well. It is therefore also consistent with neuro-computational naive realism that the subjects have perceptions with different phenomenologies. For this reason, none of the cases poses a problem to neuro-computational naive realism.

The third advantage of neuro-computational naive realism is that it supports the common sense view that perceptions and hallucinations can be phenomenally similar. The explanation is simple: A subject who is perceiving and a subject who is hallucinating can have the same neuro-computational properties. According to neuro-computational naive realism, a subject's neuro-computational properties completely determine what appearance properties she has. It follows that a subject who is perceiving and a subject who is hallucinating can have the same appearance properties. In other words, a subject who is perceiving and a subject who is hallucinating can appeared to in the same ways. For example, you can be appeared to in a roundish way in both a perception and in a hallucination. Thus, perceptions and hallucinations can be phenomenally similar.

Importantly, however, while neuro-computational naive realism supports the view that perceptions and hallucinations can be phenomenally similar, it also suggests that they are phenomenally different. The difference is that the phenomenologies of perceptions, but not of hallucinations, involve items' perceptually appearing certain ways to a subject. And for an item to perceptually appear certain ways to a subject, the subject has to perceive that item. Therefore, whenever some item perceptually appears some way to a subject, the subject is having a perception and not a hallucination. And whenever a subject is having a hallucination, nothing perceptually appears to the subject in the having of that hallucination. For example, when a ball perceptually appears round to you, you perceive the ball rather than hallucinate it. And when you hallucinate a ball, neither the ball nor anything else perceptually appears to you in the having of that hallucination. $^{27}$

\section{Will naive realists be happy with neuro-computational naive realism?}

Despite neuro-computational naive realism's advantages, I expect that some naive realists will be inclined to resist it. The naive realists I have in mind are those who endorse the following two theses ${ }^{28}$ :

\footnotetext{
27 This briefly sketched view of hallucinations must eventually face Martin's $(2004,2006)$ arguments that disjunctivists should only give negative accounts of hallucinations, i.e., ones which characterize hallucinations in terms of certain perceptions. These arguments are concerning, because my sketch might plausibly be charged of being a positive account of hallucinations, which appeals to the hallucinator's having certain appearance properties. I nonetheless offer my sketch, as I accept Logue's (2013) and Johnston's (2004, pp. 138-139) dissenting views on how disjunctivists should account for hallucinations.

${ }^{28}$ Campbell (in Campbell and Cassam 2014, p. 33), e.g., endorses (Constitution). (Inheritance) is endorsed, e.g., by Allen (2016, pp. 13-14) and Campbell (1993, p. 268).
} 
(Constitution) The phenomenologies of perceptions are constituted by mindindependent qualities of objects (such as colors, shapes and sizes).

(Inheritance) The phenomenologies of perceptions are inherited from mindindependent qualities of objects (such as colors, shapes and sizes).

Although these theses go well beyond the core commitments of naive realism [i.e., beyond (NR1)-(NR3)], naive realists who endorse them would be dissatisfied if they proved to be incompatible with neuro-computational naive realism. I close this paper with a discussion of this worry.

Start with (Constitution). Happily, this thesis is fully compatible with neurocomputational naive realism. According to neuro-computational naive realism, perceptual phenomenology is fixed by instantiations of the $x$ perceptually appears $W$ to $S$ relation. Such instantiations have perceptually appearing items $x$ as constituents. If we required some of these items to be mind-independent qualities of objects, it would follow that the phenomenologies of perceptions are constituted by mind-independent qualities of objects, just as the inheritance thesis requires. In other words, neuro-computational naive realism can retain the inheritance thesis by holding that any perception has some aspects of perceptual phenomenology in which mind-independent qualities of objects $Q$ perceptually appear to the subject.

I now turn to (Inheritance). Though this thesis is not completely clear, what it seems to suggest is that the ways that items perceptually appear to subjects are inherited from mind-independent qualities of objects. In other words, (Inheritance) seems to suggest that appearance properties - at least those appearance properties that are instantiated when things perceptually appear some way to someone-are inherited from mind-independent qualities of objects. For example, an application of (Inheritance) to a case in which a ball perceptually appears round to you would seem to amount to the suggestion that your appearance property of being appeared to in a roundish way is inherited from the mind-independent quality of roundness (which round objects have).

It is doubtful that all prominent naive realists would accept (Inheritance). In particular, Martin's (1998) view is consistent with rejecting it. It is also doubtful that (Inheritance) is true. ${ }^{29}$ So if neuro-computational naive realism proves incompatible with (Inheritance), it is unclear if that would be a serious cost.

Still, since (Inheritance) is independently interesting, I would like to explore whether neuro-computational naive realism is compatible with it. I do so by quickly sketching two versions that neuro-computational naive realism might take-an

\footnotetext{
29 An anonymous reviewer suggested to me that (Inheritance) must be weakened if it is to survive the following variant of a case originally due to David Chalmers: Suppose there are two dots right next to each other on blank field, with a cross between them. While fixing your eyes on the cross, you attend first to the dot on the right and then to the dot on the left. Since the dots are very close together, it is plausible that you perceive the properties of the dots at the same level of determinacy. Nevertheless, the empirical results related to the attentional variation case suggest that the dots would appear differently to you. The case therefore recommends a weakening of (Inheritance). The reviewer also noted that (Inheritance) might need to be weakened to accommodate cases of blurry vision. I am very grateful to the reviewer for both these suggestions. For a further discussion of blurry vision in a naive realist setting, see French (2014).
} 
"identity version", which is incompatible with (Inheritance); and a "relational version", which is compatible with (Inheritance).

The identity version suggests that appearance properties are identical to certain intrinsic neuro-computational properties. ${ }^{30}$ This raises three issues: First, intrinsic neuro-computational properties of subjects are not identical to shapes, sizes or any other mind-independent qualities of objects. Second, intrinsic neuro-computational properties cannot be relations to mind-independent qualities of objects. And finally, changes in the distribution of intrinsic neuro-computational properties of subjects and changes in the distribution of mind-independent qualities of objects can plausibly occur independently of each other. These three issues make it difficult to see how intrinsic neuro-computational properties of subjects could be inherited from mind-independent qualities of objects. So if appearance properties are identical to certain intrinsic neuro-computational properties of subjects, (Inheritance) would be false.

Alternatively, the relational version suggests that though subjects have the appearance properties that they do in virtue of their neuro-computational properties, appearance properties are distinct from neuro-computational properties. What appearance properties are identical to is certain non-perceptual relational properties. More specifically, the relational version suggests that for $S$ to have an appearance property is for $S$ to bear some non-perceptual relation to a mind independent quality $Q$, which reveals $Q$ to $S$. For example, to be appeared to in a roundish way is to bear a relation to roundness, which reveals roundness to you. Similarly, to be appeared to in a blueish way is to bear a relation to blueness, which reveals blueness to you. Nevertheless, you have the appearance properties you do in virtue of your neurocomputational properties. So, e.g., though you are related to roundness in being appeared to in a roundish way, that relationship is due to your neuro-computational properties. $^{31}$

The relational version is compatible with (Inheritance) because it seems to provide a sense in which appearance properties are inherited from mindindependent qualities of objects. For example, suppose a blue ball perceptually appears green to Alice. In this case, Alice is appeared to in a greenish way, and so is (non-perceptually) related to greenness. Greenness is thereby both revealed to Alice, and is a constituent of Alice's appearance property. This seems to provide a clear sense in which Alice's appearance property is inherited from greenness.

\footnotetext{
30 The identity version is very similar to Block's (2009), McLaughlin's (2007) and Papineau's (2014) view that phenomenal properties (which characterize aspects of what it is like to be a subject) are identical to certain intrinsic neuro-computational properties. But despite this similarity, there is also a dissimilarity between the identity version and their view: They tend to think of phenomenal properties as fixing a perception's phenomenology, whereas the identity version-like any extension of neurocomputational naive realism-insists that appearance properties are not enough to fix a perception's phenomenology.

31 Interestingly, the relational version is similar to Pautz's (2017) suggestion that a subject's neurocomputational properties ground phenomenal representation relations that the subject bears to mindindependent qualities. Note, however, that the relational version is not committed to the thought that neuro-computationally grounded relations to mind independent qualities are phenomenal representation relations.
} 
A possible concern for the relational version is its suggestion that your appearance properties are both (1) necessitated by your intrinsic neuro-computational properties, and (2) non-perceptually relate you to mind independent properties. This may sound odd. How can relations to properties be necessitated by intrinsic properties? Though an interesting question, I am not sure that it should lead us away from the relational version. This is because relations to many properties are necessitated by intrinsic properties. For example, your height is an intrinsic property, yet it necessitates you to stand in certain relations to other height properties (e.g., having a height greater than an inch). Similarly, your mass is an intrinsic property, yet it necessitates you to stand in certain extrinsic relations to other mass properties (e.g., being more massive than a gram). Granted, such examples fall short of a full explication of the relationship between neurocomputational properties and relational appearance properties. Nonetheless, the examples do show that this relationship is both coherent and worthy of further study.

In sum, both the identity version and the relational version are interesting views deserving of attention. The identity version offers an appealing physicalist reduction of appearance properties, while abandoning (Inheritance). The relational version is compatible with (Inheritance), but it is less clear whether it allows appearance properties to be reduced to physical ones. Furthermore, the relational version interestingly suggests that appearance properties involve non-perceptual relations to mind independent qualities.

I will not try to decide between the identity version and the relational version here. My goal in this paper is to propose neuro-computational naive realism as an attractive version of naive realism. I have done so by showing that neurocomputational naive realism accommodates both the internal-dependence and external-directedness of perceptions.

Acknowledgements I am indebted to Adam Pautz for the insightful discussions and criticisms that led me to write, and later improve, this paper. I have also benefitted greatly from suggestions made by Hagit Benbaji, Craig French, Anil Gupta, Ulf Hlobil, Thomas Raleigh, Assaf Weksler, David Widerker, and an anonymous reviewer. Finally, I would like to thank the participants of the ECMN Forum in Durham UK, the Serious Metaphysics Group in Cambridge UK, and the 20th conference of the Israeli Philosophy Association for valuable comments and advice.

Open Access This article is distributed under the terms of the Creative Commons Attribution 4.0 International License (http://creativecommons.org/licenses/by/4.0/), which permits unrestricted use, distribution, and reproduction in any medium, provided you give appropriate credit to the original author(s) and the source, provide a link to the Creative Commons license, and indicate if changes were made.

\section{References}

Allen, K. (2016). A naive realist theory of colour. Oxford: Oxford University Press.

Antony, L. (2011). The openness of illusions. Philosophical Issues, 21, 25-44.

Austin, J. L. (1962). Sense and sensibilia. Oxford: Oxford University Press.

Block, N. (Ed.) (2007a). Sexism, racism, ageism, and the nature of consciousness. In Consciousness, function, and representation (Vol. 1, Chap. 25, pp. 571-601). Cambridge, MA: MIT Press. 
Block, N. (2007b). Wittgenstein and qualia. Philosophical Perspectives, 21, 73-115.

Block, N. (2009). Comparing the major theories of consciousness. In M. S. Gazzaniga (Ed.), The cognitive neurosciences (4th ed., Chap. 77, pp. 1111-1122). Cambridge, MA: MIT Press.

Block, N. (2010). Attention and mental paint. Philosophical Issues, 20, 23-63.

Brewer, B. (2011). Perception and its objects. Oxford: Oxford University Press.

Brewer, B. (2013). Attention and direct realism. Analytic Philosophy, 54, 421-435.

Brouwer, G. J., \& Heeger, D. J. (2009). Decoding and reconstructing color from responses in human visual cortex. The Journal of Neuroscience, 29, 13992-14003.

Brouwer, G. J., \& Heeger, D. J. (2013). Categorical clustering of the neural representation of color. The Journal of Neuroscience, 33(39), 15454-15465.

Byrne, A., \& Hilbert, D. R. (1997). Colors and reflectances. In A. Byrne \& D. R. Hilbert (Eds.), Readings on color, (Vol. 1, Chap. 14, pp. 263-288). Cambridge, MA: MIT Press.

Campbell, J. (1993). A simple view of colour. In J. Haldane \& C. Wright (Eds.), Reality, representation and projection (Chap. 9, pp. 257-268). Oxford: Oxford University Press.

Campbell, J. (2002). Reference and consciousness. Oxford: Oxford University Press.

Campbell, J. (2009). Consciousness and reference. In B. McLaughlin, A. Beckermann, \& S. Walter (Eds.), Oxford handbook of philosophy of mind (pp. 648-662). Oxford: Oxford University Press.

Campbell, J., \& Cassam, Q. (2014). Berkeley's puzzle. Oxford: Oxford University Press.

Carrasco, M., Ling, S., \& Read, S. (2004). Attention alters appearance. Nature Neuroscience, 7, 308-313.

Chen, J.-Y., Victor, J. D., \& Di Lorenzo, P. M. (2011). Temporal coding of intensity of nacl and hcl in the nucleus of the solitary tract of the rat. Journal of Neurophysiology, 105(2), 697-711.

Conway, B. R., Chatterjee, S., Field, G. D., Horwitz, G. D., Johnson, E. N., Koida, K., et al. (2010). Advances in color science: From retina to behavior. The Journal of Neuroscience, 30(45), $14955-14963$.

Cowart, B. J., \& Rawson, N. E. (2005). Olfaction. In E. B. Goldstein (Ed.), Blackwell handbook of sensation and perception (Chap. 18, pp. 567-600). Malden, MA: Blackwell.

Di Lorenzo, P. M., Chen, J.-Y., \& Victor, J. D. (2009). Quality time: Representation of a multidimensional sensory domain through temporal coding. The Journal of Neuroscience, 29(29), 9227-9238.

Fish, W. (2009). Perception, hallucination, and illusion. Oxford: Oxford University Press.

Foster, D. H. (2011). Color constancy. Vision Research, 51, 674-700.

French, C. (2014). Naive realist perspectives on seeing blurrily. Ratio, 27, 393-413.

Genone, J. (2014). Appearance and illusion. Mind, 123, 339-376.

Genone, J. (2016). Recent work on naive realism. American Philosophical Quarterly, 53, 1-26.

Gobell, J., \& Carrasco, M. (2005). Attention alters the appearance of spatial frequency and gap size. Psychological Science, 16, 644-651.

Hardin, C. L. (1988). Color for philosophers. Indianapolis: Hackett.

Howard, J. D., Plailly, J., Grueschow, M., Haynes, J.-D., \& Gottfried, J. A. (2009). Odor quality coding and categorization in human posterior piriform cortex. Nature Neuroscience, 12, 932-938.

Hurvich, L. M., Jameson, D., \& Cohen, J. D. (1968). The experimental determination of unique green in the spectrum. Perception and Psychophysics, 4, 65-68.

Johnston, M. (2004). The obscure object of hallucination. Philosophical Studies, 120, 113-183.

Johnston, M. (2006). Better than mere knowledge? The function of sensory awareness. In T. S. Gendler \& J. Hawthorne (Eds.), Perceptual experience (pp. 260-290). Oxford: Oxford University Press.

Kalderon, M. E. (2011). Color illusion. Noûs, 45, 751-775.

Langers, D. R., van Dijk, P., Schoenmaker, E. S., \& Backes, W. H. (2007). fmri activation in relation to sound intensity and loudness. NeuroImage, 35(2), 709-718.

Ling, S., \& Carrasco, M. (2006). When sustained attention impairs perception. Naure Neuroscience, 9 , 1243-1245.

Liu, T., Abrams, J., \& Carrasco, M. (2009). Voluntary attention enhances contrast appearance. Psychological Science, 20, 354-362.

Logue, H. (2012). Why naive realism. Proceedings of the Aristotelian Society, 112, 211-237.

Logue, H. (2013). Good news for the disjunctivist about (one of) the bad cases. Philosophy and Phenomenological Research, 86, 105-133.

Logue, H. (2017). Are perceptual experiences just representations? In B. Nanay (Ed.), Current controversies in philosophy of perception (Chap. 3, pp. 43-56). New York: Routledge.

Lutze, M., Cox, N. J., Smith, V. C., \& Pokorny, J. (1990). Genetic studies of variation in rayleigh and photometric matches in normal trichromats. Vision Research, 30, 149-162. 
MacAdam, D. L. (1985). The physical basis of color specification (2nd ed., Chap. 1, pp. 1-25). Springer: Berlin.

Malnic, B., Hirono, J., Sato, T., \& Buck, L. B. (1999). Combinatorial receptor codes for odors. Cell, 96(5), 713-723.

Margot, C. (2009). A noseful of objects. Nature Neuroscience, 12, 813-814.

Marr, D. (1982). Vision: A computational investigation into the human representation and processing of visual information. San Francisco: W. H. Freeman.

Martin, M. G. F. (1998). Setting things before the mind. Royal Institute of Philosophy Supplement, 43, 157-179.

Martin, M. G. F. (2002). The transparency of experience. Mind and Language, 17, 376-425.

Martin, M. G. F. (2004). The limits of self-awareness. Philosophical Studies, 120, 37-89.

Martin, M. G. F. (2006). On being alienated. In T. S. Gendler \& J. Hawthorne (Eds.), Perceptual experience (pp. 354-410). Oxford: Oxford University Press.

Martin, M. G. F. (2010). What's in a look. In B. Nanay (Ed.), Perceiving the world (Chap. 8, pp. 160-225). Oxford: Oxford University Press.

McDowell, J. (1996). Mind and world (2nd ed.). Cambridge, MA: Harvard University Press.

McDowell, J. (2008). The disjunctive conception of experience as material for a transcendental argument. In A. Haddock \& F. Macpherson (Eds.), Disjunctivism: Perception, action, knowledge (Chap. 16, pp. 376-389). Oxford: Oxford University Press.

McLaughlin, B. P. (2007). Type materialism for phenomenal consciouness. In M. Velmans \& S. Schneider (Eds.), The Blackwell companion to consciousness (Chap. 34, pp. 431-444). Malden, MA: Blackwell.

Montagna, B., Pestilli, F., \& Carrasco, M. (2009). Attention trades off spatial acuity. Vision Research, 49, 735-745.

Moore, B. C. J. (2003). Introduction to the psychology of hearing (5th ed.). San Diego, CA: Academic Press.

Neitz, J., \& Jacobs, G. H. (1986). Polymorphism of long-wavelength cone in normal human color vision. Nature, 323, 623-625.

Neitz, M. \& Neitz, J. (1998). Molecular genetics and the biological basis of color vision. In W. G. K. Backhaus, R. Kliegl, \& J. S. Werner (Eds.), Color vision: Perspectives from different disciplines (Chap. 5, pp. 101-120). Berlin: Walter de Gruyter.

Neitz, J., Neitz, M., \& Jacobs, G. H. (1993). More than three different cone pigments among people with normal color vision. Vision Research, 33, 117-122.

Palmer, S. E. (1999). Vision science. Cambridge: MIT Press.

Papineau, D. (2014). Sensory experience and representational properties. Proceedings of the Aristotelian Society, 114, 1-33.

Pautz, A. (2010). Do theories of consciousness rest on a mistake? Philosophical Issues, 20, 333-367.

Pautz, A. (2011). Can disjunctivists explain our access to the sensible world? Philosophical Issues, 21, 384-433.

Pautz, A. (2013). Do the benefits of naive realism outweigh the costs? Comment on fish, perception, hallucination and illusion. Philosophical Studies, 163, 25-36.

Pautz, A. (2014). The real trouble with phenomenal externalism: New empirical evidence for a brainbased theory of consciousness. In R. Brown (Ed.), Consciousness inside and out: Phenomenology, neuroscience, and the nature of experience (Chap. 18, pp. 237-298). Dordrecht: Springer.

Pautz, A. (2017). Experiences are representations: An empirical argument. In B. Nanay (Ed.), Current controversies in philosophy of perception (Chap. 2, pp. 23-42). New York: Routledge.

Putnam, H. (1994). Sense, nonsense, and the senses: An inquiry into the powers of the human mind. The Journal of Philosophy, 91, 445-517.

Relkin, E. M., \& Doucet, J. R. (1997). Is loudness simply proportional to the auditory nerve spike count? The Journal of the Acoustical Society of America, 101, 2735-2740.

Roe, A. W., Chelazzi, L., Connor, C. E., Conway, B. R., Fujita, I., Gallant, J. L., et al. (2012). Toward a unified theory of visual area v4. Neuron, 74, 12-29.

Röhl, M., Kollmeier, B., \& Uppenkamp, S. (2011). Spectral loudness summation takes place in the primary auditory cortex. Human Brain Mapping, 32(9), 1483-1496.

Sakurai, T., Misaka, T., Ueno, Y., Ishiguro, M., Matsuo, S., Ishimaru, Y., et al. (2010). The human bitter taste receptor, htas2r16, discriminates slight differences in the configuration of disaccharides. Biochemical and Biophysical Research Communications, 402(4), 595-601. 
Schiffman, S. S., \& Erickson, R. P. (1971). A psychophysical model for gustatory quality. Physiology \& Behavior, 7(4), 617-633.

Shoemaker, S. (1994). Phenomenal character. Noûs, 28(1), 21-38.

Shoemaker, S. (2000). Phenomenal character revisited. Philosophy and Phenomenological Research, $60(2), 465-467$.

Shoemaker, S. (2006). On the ways things appear. In T. S. Gendler \& J. Hawthorne (Eds.), Perceptual experience (pp. 461-480). Oxford: Oxford University Press.

Smith, D. V., Van Buskirk, R. L., Travers, J. B., \& Bieber, S. L. (1983). Coding of taste stimuli by hamster brain stem neurons. Journal of Neurophysiology, 50(2), 541-558.

Snowdon, P. (1990). The objects of perceptual experience. Proceedings of the Aristotelian Society, 64, 121-150.

Stazicker, J. (2011). Attention, visual consciousness and indeterminacy. Mind and Language, 26, 156-184.

Travis, C. (2013). The silences of the senses. In Perception: Essays after frege (Chap. 1, pp. 23-58). Oxford: Oxford University Press.

Van der Heijden, A. (1993). Sweet and bitter tastes. In T. E. Acree \& R. Teranishi (Eds.), Flavor science: Sensible principles and techniques (pp. 76-115). Providence: American Chemical Society.

Walters, D. E. (1996). How are bitter and sweet tastes related? Trends in Food Science \& Technology, 7(12), 399-403.

Youngentob, S. L., Johnson, B. A., Leon, M., Sheehe, P. R., \& Kent, P. F. (2006). Predicting odorant quality perceptions from multidimensional scaling of olfactory bulb glomerular activity patterns. Behavioral Neuroscience, 120(6), 1337-1345. 